\title{
Imaging diagnosis of nonalcoholic steatohepatitis
}

\author{
Anca Smaranda Farmazon ${ }^{1}$, Alexandru Radu Mihailovici², Rodica Diaconu², \\ Daniela Carmen Neagoe ${ }^{3}$, Gabriel Nicolae lanosi ${ }^{4}$, Oana Sorina Tica ${ }^{5}$, \\ Maria Aurelia Buse ${ }^{2}$ \\ ${ }^{1}$ Internal Medicine department, University of Medicine and Pharmacy, Emergency County Hospital, \\ Craiova, Romania \\ ${ }^{2}$ Cardiology Clinic, Emergency County Hospital, Craiova, Romania \\ ${ }^{3}$ Medical semiology department, University of Medicine and Pharmacy, Craiova, Romania \\ ${ }^{4}$ Surgical semiology department, University of Medicine and Pharmacy, Craiova, Romania \\ ${ }^{5}$ Obstetrics and Gynecology department, University of Medicine and Pharmacy, Craiova, Romania
}

\begin{abstract}
Non-alcoholic fatty liver comprises several clinical entities, starting from hepatic steatosis and reaching steatohepatitis, fibrosis and liver cirrhosis; it is currently considered a major public health issue. The most important risk factors for non-alcoholic fatty liver are obesity, type II diabetes and dyslipidaemia.

At present, it is of great importance the study of non-invasive diagnostic methods to identify imaging techniques that correlate with steatohepatitis and liver fibrosis and to establish clear indications of liver biopsy in patients with non-alcoholic fatty liver.
\end{abstract}

Keywords: non-alcoholic steatohepatitis, fibrosis, ultrasound

\section{INTRODUCTION}

Non-alcoholic steatohepatitis (NASH), described for the first time by Ludwig, is part of a group of diseases known as non-alcoholic hepatopathies, in which the accumulation of fats can determine a progressive hepatic dysfunction. The characteristic of this pathology is pointed out by a large spectrum of lesions of the liver which occur in the absence of alcohol consumption and of other obvious causes of hepatic disease. It is characterised by an inflammation due to the accumulation of fat in the liver.

Non-alcoholic fatty liver disease (NAFLD) refers to the spectrum of hepatic diseases made of: different degrees of non-alcoholic hepatic steatosis (fatty liver), the non-alcoholic steatosis (NASH) and hepatic cirrhosis, NAFLD being the main cause of the so called "cryptogenic cirrhosis" (1).

It is considered as the hepatic manifestation of the metabolic syndrome, because it is strongly associated to the insulin resistance (IR), central obe- sity, reduced tolerance to glucose, type 2 diabetes mellitus, high blood pressure and hypertriglyceridemia. This represents a large spectrum of hepatic disorders, varying from simple non-alcoholic fatty liver (NAFL) to non-alcoholic steatohepatitis (NASH); these disorders are characterised not only by an inflammatory state, hepatocyte ballooning, necro-apoptosis and fibrosis but also through a relevant impact on morbidity and mortality.

There is a strong connection between the nonalcoholic fatty liver and the components of the metabolic syndrome (obesity, type 2 diabetes mellitus, dyslipidaemia), the main pathogenic syndrome being represented by insulin resistance (2). For the definition of the metabolic syndrome, according to IDF (International Diabetes Federation) and NHLBI (National Heart, Lung and Blood Institute) at least three out of five clinic features are necessary:

- abdominal circumference over the values set as limits on different geographic regions and population groups; 
- serum triglycerides over $150 \mathrm{mg} / \mathrm{dl}$ or hypertriglyceridemia treatment;

- HDL cholesterol under $40 \mathrm{mg} / \mathrm{dl}$ for men and under $50 \mathrm{mg} / \mathrm{dl}$ for women, or treatment for HDL-cholesterol;

- blood pressure over $130 / 85 \mathrm{mmHg}$ or anti high blood pressure treatment;

- fasting glycaemia over $100 \mathrm{mg} / \mathrm{dl}$ or using drugs for hyperglycemia $(3,4)$.

\section{IMAGING METHODS OF DIAGNOSIS}

A. Abdominal ultrasound - is the easiest usable technique and it is currently the most frequent screening method in asymptomatic patients who present increased levels of hepatic transaminases or suspicion for NAFLD. It represents a non-invasive, cheap and available on a large-scale method, being useful for the detection of hepatic steatosis with a $60-94 \%$ sensitivity. It is a subjective method based on the evaluation of hepatic echogenicity, of hepatic-renal contrast, of the loss of echogenicity if the vascular walls from the port system and the visualization of the diaphragm (5-7).

A recent trial elaborated and published by Palmentieri and collaborators on a group of 235 patients who were submitted to abdominal ultrasound and hepatic biopsy proved a $91 \%$ sensitivity in order to predict at least $30 \%$ of the steatosis cases. However, the presence of hepatic hyperreflectivity traced on ultrasound was not associated to the fibrosis within this trial.

Through transabdominal ultrasound we can identity the steatosis only when it exceeds $20-30 \%$, but it does not differentiate it from fibrosis. The steatosis liver is a large liver, with increased echogenicity (large, white and shiny liver), with venous pseudo dilatations, with posterior attenuation, and in case of cirrhosis with portal high blood pressure ultrasound signs (8). The sensitivity of the method is low (55\%, $49 \%$ respectively) when the accumulation of triglycerides is less than $30 \%$ or in the case of patients suffering from morbid obesity (9). The ultrasound appreciation of hepatic steatosis is a significant one, allowing a scaling of the fatty hepatic loading $(10,5,11)$ :

- degree 0: steatosis free;

- degree 1: mild steatosis - less than third of the parenchyma is affected, discreet posterior attenuation and visible diaphragm;

- degree 2: medium steatosis - less than a half of the parenchyma is affected, obvious posterior attenuation and blurred diaphragm;
- degree 3: severe steatosis - more than half of the parenchyma is affected, invisible posterior diaphragm.

The advantages of abdominal ultrasound include safety, the low cost, the repeatability, becoming thus the first intention imaging technique in the evaluation of patients suspected of non-alcoholic hepatic disease. The limits of the method consist in the low sensitivity in detecting incipient steatosis modifications, the low accuracy in the meteorised patients and in those suffering from obesity, the inter-observer variability and the impossibility to differentiate the simple steatosis from NASH (12).

The hepato-renal index is an ultrasound parameter used for the quantification of hepatic steatosis $(13,14)$, which proved in clinical trials a very good correlation with the histologic aspects (15) and with the content in triglycerides determined by spectroscopic magnetic resonance (7).

Doppler ultrasound is another technique that can be used in hepatic steatosis diagnosis, by which one studies the association between NAFLD and the perfusion of hepatic parenchyma (13). In 2011, on a group of 240 subjects ( 60 witness, 60 with $1 \mathrm{st}$ degree steatosis, 60 with 2 nd degree steatosis, 60 with 3rd degree steatosis - submitted to an ultrasound evaluation), a team of researchers investigated the relation between the degree of fatty infiltration and the maximum and medium speed in the portal vein, namely the resistivity index in the hepatic artery. In this trial, the analyzed Doppler parameters were correlated inversely proportional to the steatosis degree (16). Another Doppler parameter, used in the NAFLD evaluation, was the Doppler perfusion index which proved highly predictive in hepatic steatosis in patients with non-alcoholic fatty liver (17).

The Fatty Liver Index - FLI. Associated to the ultrasound examination, one elaborated an algorithm that takes into account: body mass index (BMI), waist circumference, serum triglycerides (normal values between 0 and 100) and serum level of gamma-glutamyl transferase (GGT). It was proposed as a method of identification of patients suffering from NAFLD (26). By comparing with ultrasound results (reference standard of the hepatic reference), FLI is an excellent diagnosis index of the fatty liver, with $84 \%$ accuracy, but with a diagnosis which is far from being perfect. Although FLI is a test which involves simple clinical parameters, was not validated for the staging of the steatosis (18).

Bedogni et al. used data from 216 patients under suspicion of hepatic disease and 280 patients free 
of suspicion of hepatic disease, belonging to the Dionysos trial (19) for in which the presence of hepatic steatosis was determined by ultrasound. In the cohort in question, a value of FLI below 30 allowed the exclusion of hepatic steatosis, and a value $\geq 60$ confirmed its presence, in comparison to the ultrasound evaluation (20).

In front of these evidences, the clinical use of FLI stands in the selection of patients under clinic and ultrasound suspicion of NAFLD on which one must continue the investigations and that must be monitored for the apparition of metabolic complications.

Another index with potential of identification of the hepatic steatosis is LAP (lipid accumulation product). It uses two variables, the abdominal circumference and the value of the serum triglycerides, being initially developed on the data of the American patients from NHANES III (United States' National Health and Nutrition Examination Survey) (21). The utility of the index is limited to the identification of the patients that require a specialty consultation for the diagnosis of the non-alcoholic fatty liver disease, being however necessary to the subsequent evaluation and in other population groups (22).

The abdominal ultrasound with contrast agent - using Sonoview, is the imaging technique of great value in the differentiation of the simple steatosis form Steatohepatitis, but it remains presently only an experimental technique, not being used in the current practice.

\section{Tests used to determine the steatosis, fibrosis and hepatic inflammation}

1. SteatoTest - is a quantitative test which estimates the hepatic steatosis, which comprises more parameters: age, sex, body mass index (BMI), serum levels of ALT, GGT, total bilirubin, glycaemia, cholesterol, triglycerides, $\alpha 2$-macroglobulin, apolipoprotein A1, haptoglobin. SteatoTest has a $90 \%$ sensitivity and specificity in predicting the steatosis (5).

SteatoTest offers a quantitative estimation of the hepatic steatosis, having a greater value in the steatosis diagnosis than the classic markers of hepatosteatosis (gamma-glutamyl transpeptidase and GPT) (23). SteatoTest involves several serologic parameters: the total cholesterol, the triglycerides, the glycaemia, GPT, GOT, total bilirubin, haptoglobin, gamma-glutamyl transpeptidase, $\alpha 2$ macroglobulin, apolipoprotein A1. In the healthy subjects the value of the test is of $0.14,0-0.37$ in the absence of the steatosis, $0.38-0.56$ in the 1 st degree steatosis $(1-5 \%), 0.57-0.68$ for the 2 nd degree steatosis (5$33 \%)$ and $0.69-1$ for the $3 \mathrm{rd}$ degree $(34-66 \%)$ of steatosis (23).

2. NashTest - conceived by BioPredictive it is a commercial test validated for the evaluation of steatohepatitis in patients with non-alcoholic hepatic disease and it includes a series of parameters such as: total bilirubin, GGT, $\alpha 2$-macroglobulin, apolipoprotein A1, haptoglobin and ALT, and it adjusted for age and gender, adding the weight and height. NashTest must be performed only in the patients with positive SteatoTest. Combine with FibroTest, NashTest proved its value in the screening for NASH in patients with metabolic risk. NashTest divides the patients with NASH diagnosis into three categories, in concordance with the histologic NASH score: "NASH", "NASH probable" and "NASH absence". In the validation trial performed on 257 patients, the NASH Test values for the three categories were 0.75 , 0.5 and 0.25 respectively. In order to facilitate the visual interpretation, the result is mandatorily accompanied by a graphic in colors on three classes of severity: green - NASH free, orange - NASH probable, red - NASH certain (Fig. 1) (23).
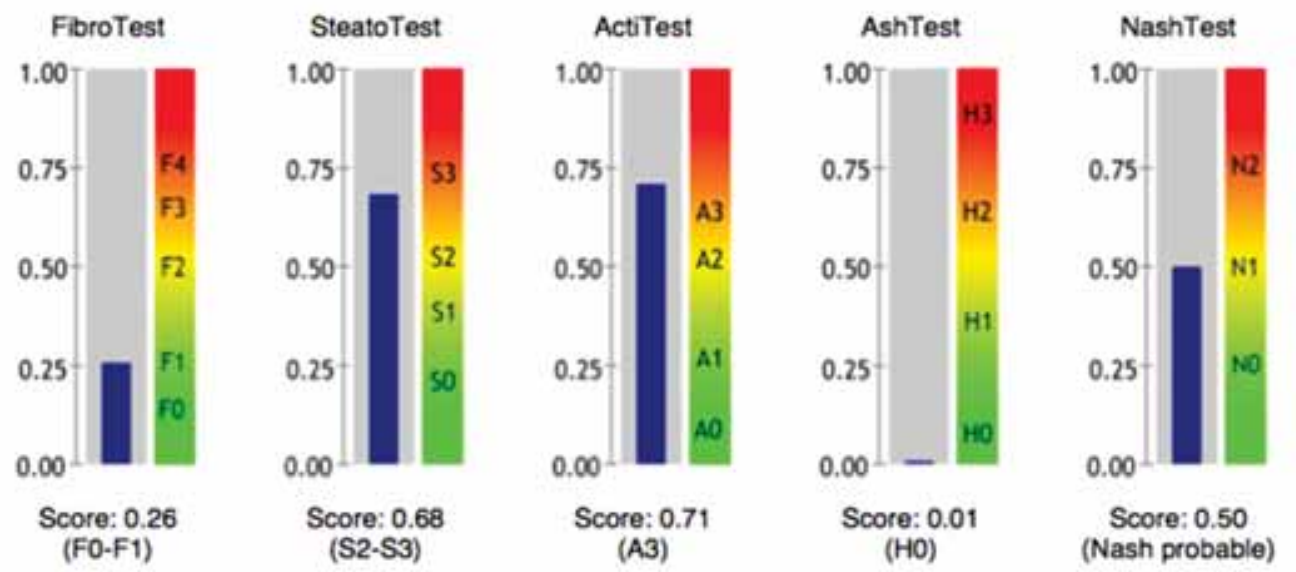

FIGURE 1. Comparison of different tests for steatosis, fibrosis and inflammation 
3. FibroTest - represents a non-invasive alternative to the hepatic biopsy ("the gold-standard" for the evaluation of the histologic lesions of the liver), which was clinically validated in patients suffering from $\mathrm{B}$ and $\mathrm{C}$ chronic hepatitis, ethanol induced hepatitis and non-alcoholic hepatic steatosis. It is based upon an algorithm that combines the results obtained in the determination of some serum biochemical markers in order to evaluate the fibrosis degree and the necro-inflammatory activity. Thus the Fibro Test measures the fibrosis degree by combining the following markers: alpha-2 macroglobulin, haptoglobin, apolipoprotein $\mathrm{A} 1$, total bilirubin, GGT, and AT estimates the activity degree (necrosis and inflammation) by associating the alanine aminotransferase to the above markers. The FibroTest-ul presents the same diagnosis value both for the intermediary stages and also for the extreme stages of fibrosis (24). The result of the FibroTest is a core from 0 to 1 , proportional to the severity of the fibrosis, being presented in a form similar to the METAVIR classification (from F0 to F4) accompanied also by a graphic on three severity classes (green - minimum or absent, orange moderate, red - significant) (Fig. 2) (23).

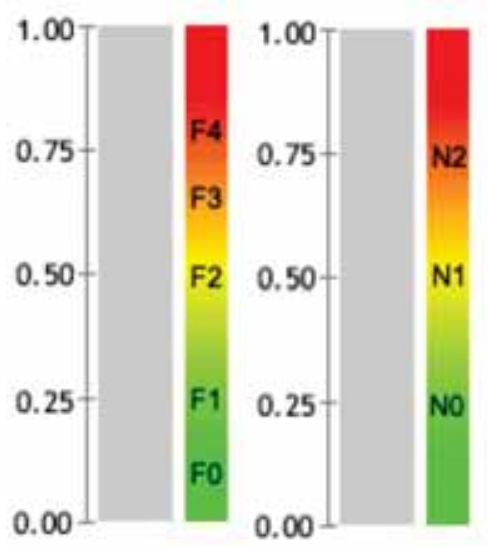

FIGURE 2. FibroTest

There is a series of limits related to the errored interpretation of the results in approximately $5 \%$ of the cases. The most frequent cause of false negative results is given by the increased levels of haptoglobin in the acute inflammations or sepsis. The bilirubin also can be increase in the hemolytic syndromes.

In 2009, Kotronen and his team of Finnish researchers developed a score - NAFLD liver fat score - that allows the determination of non-alcoholic steatosis by using clinical data a usual laboratory data: the presence of diabetes and of metabolic syndrome, basal insulinemia, serum AST level and the AST/ALT ratio, each of these parameters being associated to the presence of steatosis. Values of the score over -0.640 are predictive for non-alcoholic steatosis with an $86 \%$ sensitivity and a $71 \%$ specificity.

Advanced fibrosis in patients suffering from NAFLD is associated to the level of the serum markers of the fibrogenesis: the propeptide of the collagen type III, the hyaluronic acid, the tissue inhibitor of the matrix-metalloproteinase-1; these markers have been combined in a numeric score Enhanced Liver Fibrosis (ELF), a value exceeding 0.3576 having a $80 \%$ sensitivity and a $90 \%$ specificity for the severity of the fibrosis in NAFLD. In the same study in 2008, the authors combine NAFLD fibrosis score to the ELF score, more than 91 patients being tested and obtaining a better performance than each score taken separately $(91 \%$ sensitivity, 96\% specificity) (25).

The FibroTest, the ELF score and the combined score received only an internal validation in the original trials, that is why the reproducibility in different populational groups is unknown (25).

3. NAFLD fibrosis score - comprises 6 variables: age, hyperglycaemia, BMI, the number of platelets, the albumin and the AST/ALT ratio. The validation test was made on two groups: a group of 480 patients suffering from NAFLD confirmed by biopsy, and the control group made of 253 patients. For the two groups the positive predictive values were $90 \%, 82 \%$ respectively for the advanced fibrosis, with the cut-off value of 0.676 . For the cutoff of -1.455 the negative predictive values were $93 \%$, and $88 \%$ respectively. Between the two limits the score is considered undetermined, thorough investigations being necessary for the classification of the patients $(26,9)$. Subsequently the score has received an external validation also in 12 trials, including two multicentric trials from Japan and the United States, 3064 patients being enrolled (25). NAFLD fibrosis score can be easily calculated with routine clinical parameters, it has a good diagnosis performance, being able to be used in combination with other non-invasive tests in order to reduce the number of the patients that need a hepatic biopsy (25).

Other evolution scores have been proposed, most of them being useful for the exclusion of the advanced fibrosis (26) (Table 1).

4. FibroScan: Impulse elastography (FibroScan) is a non-invasive method that measures the tissue elasticity using a transducer which emits waves of low frequency and amplitude, which will be reflected differently by the hepatic parenchyma, depending on the hardness (the fibrosis degree) and 
TABLE 1. Non-invasive evaluation of hepatic fibrosis

\begin{tabular}{|l|l|}
\hline Test & Characteristics \\
\hline AST/ALT ra o (AAR) & $\begin{array}{l}\text { Cut-off } 0.8 \\
\text { Se } 74 \% \text { Sp } 78 \% \\
\text { VPN 93\% for the advanced fibrosis diagnosis }\end{array}$ \\
\hline APRI (AST-to-platelet ra o index) & $\begin{array}{l}\text { Developed for the viral chronic hepa } \mathrm{s} \text { C } \\
\text { Nonspecific for NAFLD }\end{array}$ \\
\hline BAAT & Score 0 or 1 VPN $100 \%$ for the septal fibrosis/cirrhosis \\
\hline BARD & $\begin{array}{l}\text { Score } 2-4 \text { predic ve for advanced fibrosis } \\
\text { VPN } 96 \% \text { for the exclusion of the advanced fibrosis }\end{array}$ \\
\hline FIB-4 & $\begin{array}{l}\text { Cut-off }<1.3-\text { absence of advanced fibrosis } \\
\text { Cut-off }>2.67-\text { presence of advanced fibrosis } \\
\text { VPP } 80 \%, \text { VPN } 90 \%\end{array}$ \\
\hline VAI (visceral adiposity index) + HOMA-IR & $\begin{array}{l}\text { VPP } 83 \% \text { for VAI }>2.1 \text { and HOMA }>3.4 \\
\text { VPN } 74 \% \text { for VAI } \leq 2.1 \text { and HOMA } \leq 3.4\end{array}$ \\
\hline
\end{tabular}

recaptured by the exploratory probe. The propagation speed depends on hepatic elasticity, the estimation of elasticity being expressed in kilopascals. The devices evaluate a tissue volume of approximately 100 times bigger than the one collected through with a $4 \mathrm{~cm}$ length and $1 \mathrm{~cm}$ diameter. The method is completely painless, reproducible, and the result is obtained and interpreted on the spot. Fibroscan is difficult to perform in obesity, in patients suffering from ascites, constitutionally narrow intercostal spaces or it is formally counterindicated during pregnancy. Initially validated and introduced in guidelines for viral chronic hepatitis $\mathrm{C}$, the $\mathrm{HIV} / \mathrm{HCV}$ coinfection and the cholestatic hepatic diseases, the method was validated also in the non-alcoholic fatty liver disease. In the metanalysis from 2011, Musso proposes more diagnosis algorithms that combine the Fibroscan to other non-invasive methods and hepatic biopsy for the evaluation of patients under suspicion of NAFLD (Fig. 3) (25).

Real time ultrasound elastography - estimates the axial hardness of the tissues along the direction of the ultrasound and displays this infor- mation as real time color images. Used for the noninvasive evaluation of the fibrosis in the viral chronic hepatitis, the obtained results are controversial since the method is a semi-quantitative one, requiring additional trials for the establishment of the value of the method in the non-invasive differentiation of the hepatic fibrosis stages.

Acoustic Radiation Force Impulse (ARFI) Elastography - represents another elastography technique for which the preliminary trials have showed results comparable to those of the ultrasound elastography in patients with hepatic cirrhosis, but less sensitive for the determination of the mild or moderate fibrosis.

B. Computed tomography is the imaging method useful in hepatic steatosis and it is characterised by the decrease in the density of hepatic parenchyma due to the strong connection between the lipid content and the hepatic attenuation - the hepatic parenchyma seems to have a darker color ("large and grey" liver), and the hyperdense vessels and bile ducts. To evaluate hepatic steatosis, one registers the absolute value of the attenuation, measured in Hounsfield units (HU), the attenuation dif-

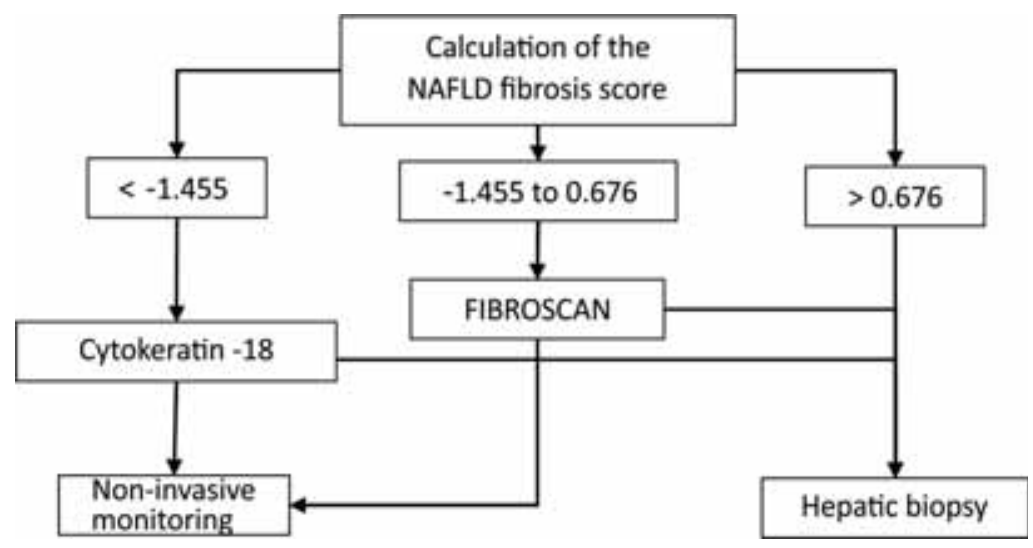

FIGURE 3. Diagnosis algorithm in NAFLD (25). 
ference between the liver and the spleen and the ratio of the two values. In the case of healthy liver, the attenuation value is of 50-57 HU (Hounsfield units), 8-10 times greater than the one of the spleen. Values lower than $40 \mathrm{HU}$ or $10 \mathrm{HU}$ times lower than the spleen ones are highly predictive for hepatic steatosis. Also, a liver/spleen attenuation ratio greater than 1.1 is highly suggestive for the moderate steatosis when it exceeds $10 \%$ and it has an 85 $90 \%$ sensitivity, reaching $93 \%$ when the steatosis exceeds $33 \%$, with a positive predictive value of $76 \%(8,9)$. The computed tomography is not an election method in the evaluation of the hepatic fibrosis, being a method with exposure to radiations (12).

C. The spin-eco type magnetic resonance is a rarely used method in the identification and evaluation of steatosis, but the spectroscopic method ofmetabolism by dosing the ATP quantity and even the one of the lipid peroxidation. These imaging methods are not usually used due to the exposure to radiations and since the two methods do not differentiate NAFLD from NASH $(5,27)$.

Spectroscopic MRI ( $\left.{ }^{1} \mathrm{H}-\mathrm{MRS}\right)$ is a non-invasive method to measure the hepatic content in lipfers the possibility of evaluating hepatic energetic

ids. Because the values obtained at ${ }^{1} \mathrm{H}-\mathrm{MRS}$ are correlated to the results of the hepatic biopsy, nowadays it is considered the optimal method of diagnosis of the hepatic steatosis (28). The first results in this sense have been obtained by Longo et al. (29-31) on subjects suffering from NAFLD on which the percent of the hepatic lipids determined by spectroscopic MRI, was correlated to the values obtained at the CT and the hepatic biopsy. By using the spectroscopic method, NAFLD is defined at a value $\geq 55.6 \mathrm{mg}$ triglycerides/gram of hepatic tissue or $\geq 5.56 \%$ of the hepatic weight.

\section{CONCLUSIONS}

NAFLD is now confirmed as the most common liver disease in the Western world, particularly due to considerable increase in metabolic diseases such as obesity and diabetes. NAFLD leads to morbidity related to liver and mortality in a subgroup of people, especially those who are obese, diabetic and who have NASH. However, a better understanding of NAFLD's natural history will allow better identification of patients at risk who need to be targeted for long-term and potentially expensive treatment.

\section{Conflict of interest: none declared Financial support: none declared}

\section{REFERENCES}

1. Clark J.M., Diehl A.M. Nonalcoholic fatty liver disease: an underrecognized cause of cryptogenic cirrhosis JAMA. 2003 Jun $11 ; 289(22): 3000-4$

2. Giovanni T., Gennaro S., Paolo C., Adriano A. Non-alcoholic Fatty Liver Disease: Further Expression of the Metabolic Syndrome, JGastroenterolHepatol 2007 Mar; 22(3):293-303.

3. Alberti et al. Harmonizing the Metabolic Syndrome A Joint Interim Statement of the International Diabetes Federation Task Force on Epidemiology and Prevention; National Heart, Lung, and Blood Institute; American Heart Association; World Heart Federation; International Atherosclerosis Society; and International Association for the Study of Obesity; Circulation. 2009;120:1640-1645.

4. Musso G., Gambino R., Bo S. et al. Should nonalcoholic fatty liver disease be included in the definition of metabolic syndrome? A cross-sectional comparison with ATP III criteria in non-obese non-diabetic subjects. Diabetes Care 2008; 31: 562-8.

5. Mohammad K.Ti., Manouchehr Kn., Shohreh Si., Parnaz Dd., Javad Ji., Masoud Gn, Zahra Si. Hepatic Artery and Portal Vein Doppler Indexes in Non-alcoholic Fatty Liver Disease Before and After Treatment to Prevent Unnecessary Health Care Costs; Int J Prev Med. 2014 Apr; 5(4): 472-477.

6. Cales P., Laine F., Boursier J. et al. Comparison of blood tests for liver fibrosis specific or not to NAFLD. J Hepatol 2009; 50: 165-73.

7. Wai C.T., Greenson J.K., Fontana R.J. et al. A simple noninvasive index can predict both significant fibrosis and cirrhosis in patients with chronic hepatitis C. Hepatology 2003; 38: 518-26.

8. Clark J.M. The epidemiology of nonalcoholic fatty liver disease in adults. J Clin Gastroenterol. 2006; 40(Suppl 1):S5-S10.

9. Angulo P. Nonalcoholic fatty liver disease in Mayo Clinic: Gastroenterology and Hepatology board review, 2008;407-418.
10. Foster K.J., Dewbury K.C., Griffith A.H., Wright R. The accuracy of ultrasound in the detection of fatty infiltration of the liver. $\mathrm{Br} \mathrm{J}$ Radiol 1980;53:440-442.

11. Kotronen A., Peltonen M., Hakkarainen A., Sevastianova K., Bergholm R., Johansson L.M., Lundbom N., Rissanen A., Ridderstråle M., Groop L., Orho-Melander M., Yki-Järvinen H. Prediction of nonalcoholic fatty liver disease and liver fat using metabolic and genetic factors; Gastroenterology 2009; 137:865-872.

12. Angulo P. GI epidemiology: Nonalcoholic fatty liver disease. Aliment PharmacolTher 2007;25:883-9.

13. Ratziu V., Giral P., Charlotte F. et al. Liver fibrosis in overweight patients. Gastroenterology 2000;118:1117-23.

14. Petta S., Amato M.C., Di Marco V., Cammà C., Pizzolanti G., Barcellona M.R., Cabibi D., Galluzzo A., Sinagra D., Giordano C., Craxì A. Visceral adiposity index is associated with significant fibrosis in patients with non-alcoholic fatty liver disease Aliment PharmacolTher. 2012 Jan; 35(2):238-47.

15. Harrison S.A., Oliver D., Arnold H.L., Gogia S., Neuschwander-Tetri B.A. Development and validation of a simple NAFLD clinical scoring system for identifying patients without advanced disease. Gut 2008; 57:1441-7.

16. Castera L., Forns X., Alberti A. Non-invasive evaluation of liver fibrosis using transient elastography. J Hepatol. 2008;48:835-847.

17. EASL Clinical Practice Guidelines: Management of hepatitis $C$ virus infection Journal of Hepatology 2011 vol. 55 j 245-264.

18. Ming-Feng Xia, Hong-Mei Yan, Wan-Yuan He, Xiao-Ming Li, Chao-Lun Li, Xiu-Zhong Yao, Ruo-Kun Li, Meng-Su Zeng, Xin Gao. Standardized Ultrasound Hepatic/Renal Ratio and Hepatic Attenuation Rate to Quantify Liver Fat Content: An Improvement Method, Obesity (Silver Spring). 2012 Feb; 20(2): 444-452. 
19. de Alwis N.M.W., Day C.P. Non-alcoholic fatty liver disease: The mist gradually clears. J Hepatol 48 (2008) S104-112.

20. Springer F., Machann J., Claussen C.D., Schick F., Schwenzer N.F. Liver fat content determined by magnetic resonance imaging and spectroscopy World J Gastroenterol 2010 April 7; 16(13): 1560-1566.

21. Kahn H.S. The "lipid accumulation product" performs better than the body mass index for recognizing cardiovascular risk: a populationbased comparison. BMC CardiovascDisord 2005, 5:26.

22. Bedogni G., Henry S. Kahn, Stefano Bellentani, Claudio Tiribelli. A simple index of lipid overaccumulation is a good marker of liver steatosis; BMC Gastroenterology 2010;10(98).

23. Mona M., Vlad R., Rachel M., Djamila M., Francoise I. Thierry Poynard Noninvasive Biomarkers for the Screening of Fibrosis, Steatosis and Steatohepatitis in Patients with Metabolic Risk Factors: FibroTest-FibroMax ${ }^{\mathrm{TM}}$ Experience J Gastrointestin Liver Dis June 2008 Vol.17 No 2, 187-191.

24. Jacqueminet S., Lebray P., Morra R. et al. Screening for liver fibrosis by using a noninvasive biomarker in patients with diabetes. ClinGastroenterolHepatol 2008, in press.

25. Goritsas C., Spanos K., Stefanopoulos D. et al. Non Alcoholic fatty liver disease: Correlation With Clinical and Biochemical Parameters. Hospital Chronical 2007,2(3):100-103.
26. Dowman J.K., Tomlinson J.W., Newsome P.N. Systematic Review: The Diagnosis and Staging of Non-alcoholic Fatty Liver Disese and Non-alcoholic Steatohepatitis. Aliment Pharmacol Ther. 2011:33(5):525-540.

27. Afshin Mohammadi, Ali Bazazi, Tooraj Maleki-Miyandoab, Mohammad Ghasemi-rad. Evaluation of relationship between Grading of fatty liver and severity of Atherosclerotic finding; Int J Clin Exp Med. 2012; 5(3): 251-256.

28. Szczepaniak L. et al. Measurement of intracellular triglyceride stores by $\mathrm{H}$ spectroscopy: validation in vivo. Am J PhysiolEndocrinolMetab 1999, 276:E977-E989.

29. Sanjeev R.M., E. Louise Thomas, Jimmy D.B., Desmond G.J., Simon D. T-R. Non-invasive means of measuring hepatic fat content World $J$ Gastroenterol 2008 June 14; 14(22): 3476-3483.

30. Longo R., Ricci C., Masutti F., Vidimari R., Croce L.S., Bercich L., Tiribelli C., Dalla Palma L. Fatty infiltration of the liver. Quantification by $1 \mathrm{H}$ localized magnetic resonance spectroscopy and comparison with computed tomography. Invest Radiol 1993; 28: 297-302.

31. Longo R., Pollesello P., Ricci C., Masutti F., Kvam B.J., Bercich L., Croce L.S., Grigolato P., Paoletti S., de Bernard B. Proton MR spectroscopy in quantitative in vivo determination of fat content in human liver steatosis. J Magn Reson Imaging 1995; 5:281-285. 